\title{
Emotional analysis of safeness and risk perception of drones during the COVID-19 pandemic in Italy
}

\author{
Fabio Garzia \\ Safety \& Security Engineering \\ Group - DICMA \\ SAPIENZA - University of Rome \\ Rome, Italy \\ \& \\ Wessex Institute of Technology \\ Southampton, UK \\ $\&$ \\ European Academy of Sciences \\ and Arts \\ Salzburg, Austria \\ fabio.garzia@uniroma1.it
}

\author{
Francesco Borghini \\ Safety \& Security Engineering \\ Group - DICMA \\ SAPIENZA - University of Rome \\ Rome, Italy \\ Mara Lombardi \\ Safety \& Security Engineering \\ Group - DICMA \\ SAPIENZA - University of Rome \\ Rome, Italy
}

\author{
Antonello Castagnolo \\ Safety \& Security Engineering \\ Group -DICMA \\ SAPIENZA - University of Rome \\ Rome, Italy \\ Soodamani Ramalingam \\ School of Engineering and \\ Computer Sciences \\ University of Hertfordshire \\ Hatfield, UK
}

\begin{abstract}
The estimation of perceived safeness and risk by individuals is very significant for safety and security managing. Every person is based on the judgments of other persons to do a choosing and the Internet personifies the location where these judgments are mostly tracked, obtained, and assessed. From this point of view, social networks have a significant influence. For this reason, Opinion Mining and Sentiment Analysis have found remarkable utilizes in a multiplicity of circumstances and one of the most notable is represented by public safety and security.

The purpose of the present work is to investigate the perception of safeness and risk of drones during COVID19 pandemic in Italy. It was considered the period from Aprile 15 to December 10, 2020, properly divided into 4 significant phases, highlighting the emotional components using the semantic analysis of the textual contents present in Twitter.
\end{abstract}

Keywords-Perceived risk assessment for security \& safety, Open-Source Intelligent Techniques for security \& safety, OSINT, opinion mining for security \& safety, sentiment analysis for security \& safety, COVID-19 pandemic, drones security \& safety.

\section{INTRODUCTION}

The estimation of perceived safeness and risk by individuals is very significant for security and safety managing.

Every person is based on the judgments of other persons to do a choosing and the Internet personifies the location where these judgments are mostly tracked, obtained, and assessed. From this point of view, social networks have a significant influence. For this reason, Opinion Mining and Sentiment Analysis have found utilizes in a multiplicity of circumstances and one of the most notable is represented by public safety and security.
Security and safety managers can collect people's perception to notice the unpredicted faults of a certain context or at least the security, safeness, and risk perception of people that sometime can be rather different from physical level of security, safeness, and risk of a certain context. This can represent a considerable way to detect continuously judgments about risk perception and when an appropriate action aimed at diminishing effective or perceived risk is done, so that it is achievable get the impact on the perceived risk [1 - 16].

The purpose of the present work is to investigate the perception of safeness and risk of drones during COVID-19 pandemic in Italy. It was considered the period from Aprile 15 to December 10, 2020, properly divided into 4 significant phases, highlighting the emotional components using the semantic analysis of the textual contents present in Twitter. The gathering of opinions to be employed for this intent involves investigating a variety of open sources (OSINT - OpenSource INTelligence) and subsequently the handling of significant quantities of data (Big Data) in digital form from which to gain information and knowledge. In our case, Twitter was employed as a supplier and a proper analysis of the keywords contained in the various tweets was done. The keywords have been selected considering that the evaluation of perceived risk is profoundly associated to the psychological features, through the emotional reactions generated by a certain context.

\section{DRONES SURVEILLANCE DURING COVID-19 PANDEMIC}

Drones were used extensively during the COVID19 pandemic around the world, including Italy. 
There have been many striking episodes of monitoring people with drones during this period in Italy and around the world.

In Italy the use of drones is regulated by ENAC (Italian Civil Aviation Authority) which, at the end of March 2020, significantly expanded the scope of use of drones to ensure the containment of the coronavirus epidemiological emergency.

This has also allowed the local police to be able to monitor the movements of citizens in the municipal area from above, even in the urban area, with drones of considerable size and weight, up to 25 kilograms.

The use of drones has made it possible to carry out filming and operations that were earlier or impossible or that would have required the use of a helicopter but also causes unprecedented forms of incursion in the private sphere of people and confidentiality and protection of their personal data. A drone patrol, intuitively, offers a completely different view, making it possible to inspect otherwise private areas (from gardens to terraces), places often typical of the concept of 'private residence', which enjoys enhanced protection according to the Italian law.

The reduction of one's private sphere due to the sanitary emergency, together with the risk of possible non-respect of privacy according to the Regulation (EU) 2016/679 (General Data Protection Regulation), has led to a non-positive perception of the use of drones, as it is demonstrated in the following.

\section{THE METHODOLOGY UTILIZED}

The semantic information comprised in the data can be achieved by means of the Sentiment Analysis, even well-recognized as Opinion Mining. This assessment takes the personal opinions of the users on an objective of interest by the estimation of the polarity of the feeling (which can be positive, negative, or neutral) employed to describe the experience with the latter. Normally, the Sentiment Analysis is staged following the distinct phases indicated in Fig.1.

The chosen data source is represented by Twitter where the texts are attained. It embodies one of the most utilized social networks in the world since it has one of the largest percentages of public profiles with respect to others (Facebook ${ }^{\circledR}$ profiles and comments are generally not public). Additionally, the text is the key component (Instagram ${ }^{\circledR}$ comprises mainly photos) whose conciseness, 280 characters / tweet, allows to review each tweet more simply, rapidly, and efficiently.

There is much software offered for social network valuation, but NodeXL is frequently the most frequent since it does not necessitate the knowledge of any programming language. NodeXL is a free plug-in for Excel ${ }^{\circledR}$ and the essential edition permits to import data and process through a proper metrics. Due to the restrictions of Twitter API, it is possible to attain up to 2000 tweets or at least tweets no older than 7-8 days in the free of charge version, but these restrictions decrease employing the pro version. Since the present work required a considerable number of tweets to be gathered and treated, the pro version was employed. In the particular case, the metric 'Words and word pairs' has been utilized. This particular metrics calculates the words and the pairs of words which are included in the tweets. Essentially, this metric gets the Sentiment Analysis by means of text scanning, identifying the words which fit in the two 'Lists of Sentiment Words' with the associated number of occurrences.

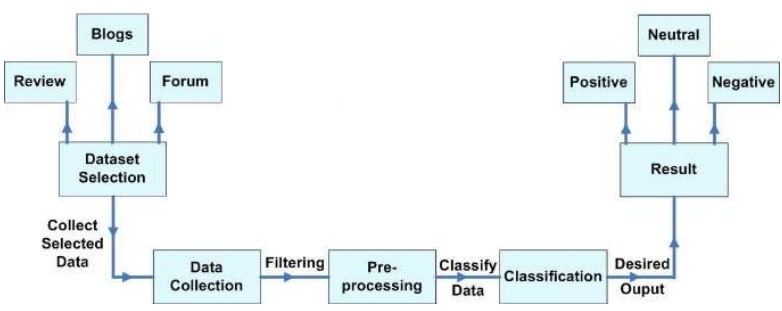

Fig. 1. General opinion mining steps.

The analysis focused on the investigation of the tweets during COVID-19 pandemic in Italy. It was considered the period from Aprile 15 to December 10, 2020, properly divided into 4 significant phases, highlighting the emotional components using the semantic analysis of the textual contents present in Twitter.

During the above analysed periods, four distinct phases have been identified and studied. They are shown in Tab. I

TABLE I. PHASES AND DESCRIPTIONS.

\begin{tabular}{|c|l|l|}
\hline Phase & \multicolumn{1}{|c|}{ Period } & \multicolumn{1}{c|}{ Description } \\
\hline 1 & $\begin{array}{l}\text { From the begin of } \\
\text { pandemic to } 31^{\text {st }} \\
\text { May 2020. }\end{array}$ & $\begin{array}{l}\text { All Italy was classified red zone. } \\
\text { There was a lockdown where and } \\
\text { the movements were limited. }\end{array}$ \\
2 & $\begin{array}{l}\text { From } 1^{\text {st }} \text { June 2020 } \\
\text { to } 30^{\text {th }} \text { September } \\
2020 .\end{array}$ & $\begin{array}{l}\text { Partial normality was re- } \\
\text { established except for limited entry } \\
\text { in certain locations and use of face } \\
\text { masks in closed spaces. }\end{array}$ \\
\hline 3 & $\begin{array}{l}\text { From } 1^{\text {st }} \text { October } \\
\text { October 2020. } 31^{\text {st }}\end{array}$ & $\begin{array}{l}\text { Worsening of the epidemic. The } \\
\text { closures start again, but only in } \\
\text { certain zones }\end{array}$ \\
\hline 4 & $\begin{array}{l}\text { From } 1^{\text {st }} \text { November } \\
2020 \text { till the end of } \\
\text { data collecting } \\
\text { period } \\
\text { December 2020) }\end{array}$ & $\begin{array}{l}\text { The catering activities, sports } \\
\text { centres and more generally } \\
\text { aggregation centres are closed. } \\
\text { Towards mid-December, Italy was } \\
\text { in semi-lockdown. }\end{array}$ \\
\hline
\end{tabular}

The Italian keywords used as a search term, after a proper selection, are represented by 'controllo droni (OR) droni polizia (OR) sicurezza droni (OR) droni emergenza (OR) droni virus (OR) droni paura (OR) droni Stato' (in English: 'drones control (OR) drone police (OR) drones security (OR) drones emergency (OR) drones virus (OR) drones fear (OR) drones State').

After a proper screening and filtering of the obtained texts, so that remains only texts pertinent to the purpose, appropriate lists in English and Italian are created. They are correlated to positive words (list\#1), negative words (list\#2) and words linked to Covid-19 
(list\#3). All the other words not matching the above lists are incorporated in the non-categorized words section.

To guarantee a significant result of the evaluation, the word list, that classes the categorization in one of the three groups, is made step by step, confiding on the default English and Italian settings of the software. It is recognized that dictionaries for the English and Italian language appropriate for this sort of evaluation are already attainable but, since some words are specific to the context, a new improved lexicon of sentiments was created.

Later, to achieve the emotional reactions of people, every word (positive or negative) was associated with one of the 8 primary emotions stimulated by it. Since it is not imaginable to consider all the words of all the lists, only the first 5 more recurring words for each of the four periods were selected. The list of the 5 selected words for the 4 considered periods, translated in English, are shown in Tab. II.

TABLE II. WORDS SELECTED FOR THE 4 PHASES

\begin{tabular}{|l|l|l|l|}
\hline \multicolumn{1}{|c|}{ Phase 1 } & \multicolumn{1}{|c|}{ Phase 2 } & \multicolumn{1}{c|}{ Phase 3 } & \multicolumn{1}{c|}{ Phase 4 } \\
\hline Drones & Drones & Lockdown & Government \\
\hline Police & State & Emergency & Swindlers \\
\hline Helicopter & Italians & Communications & TSO \\
\hline Control & Virus & Fear & Delegitimized \\
\hline Safeness & Army & Police & Threaten \\
\hline
\end{tabular}

where TSO related to phase 4 is an acronym that stands for 'Trattamento Sanitario Obligatorio' (in English: 'mandatory medical treatment') which represents a series of health interventions that can be applied in case of justified need and urgency, and if there is refusal to treatment by the subject who must receive assistance. The treatment is ordered by a provision of the Mayor, as the highest health authority of the Municipality of residence or of the Municipality where the person is temporarily, following a motivated proposal from two doctors (of which at least one belongs to the Local Health Authority of territorial jurisdiction).

To reach the goal of emotion analysis, proper surveys were created and filled by a randomly collected statistical reference sample consisting of 42 people, uniformly split between male and female, aged between 18 and 70. People were queried first the positive or negative valence of every word of the survey and then the linked emotion aroused by it, choosing between the 8 essential ones. After the results of the surveys were gathered and assessed, it was possible to get the predominant emotion connected to every word.

\section{RESULTS}

The distribution of the number of tweets and retweets across the considered timeline, split into the 4 considered phases, is shown in Fig.2.

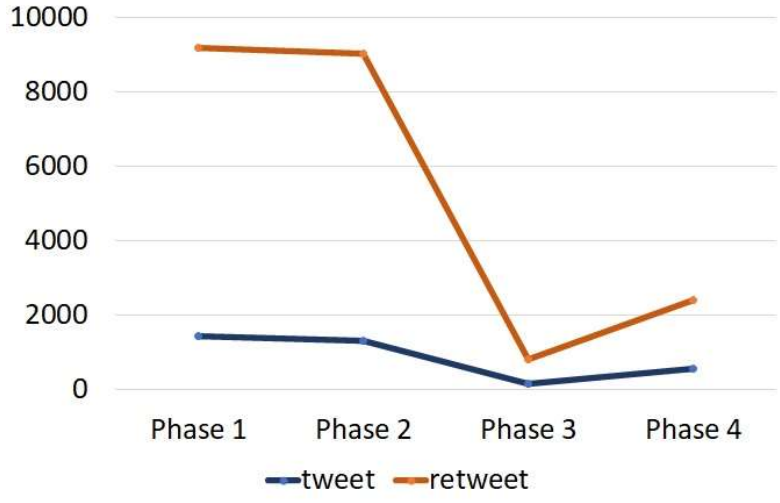

Fig. 2. Timeline of tweets and retweets for the 4 individuated phases.

Regarding phase 1, the distribution of every primary emotion is shown in Fig.3, while the positive/negative ranking of the most cited words is shown in Fig.4.

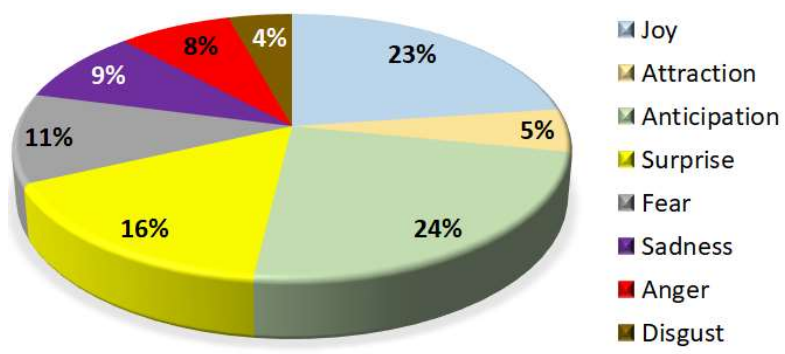

Fig. 3. Percentage distribution of every primary emotion for phase

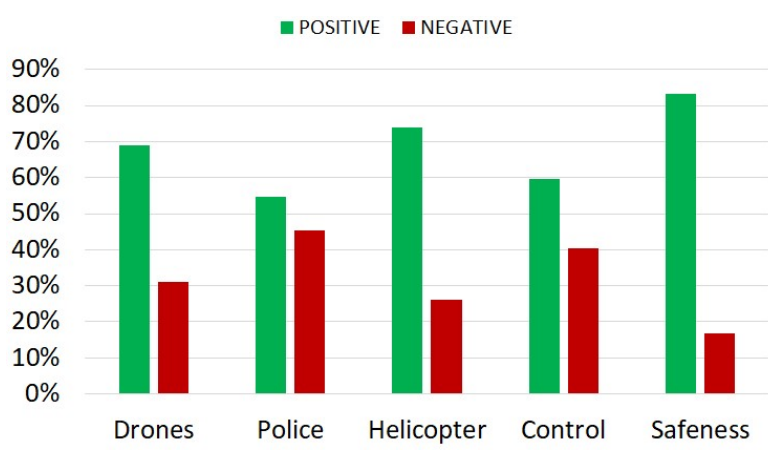

Fig. 4. Positive/negative ranking of the most cited Italian words (translated in English) for phase 1.

As it is possible to see from Fig.3, the dominant emotion occurring from the perception of safeness and risk of drones during Phase 1 of the Coronavirus pandemic is 'Anticipation' (24\%), followed by 'Joy' (23\%), 'Surprise' (16\%), 'Fear' (11\%), 'Sadness' (9\%), 'Anger' (8\%), 'Attraction' (5\%), and 'Disgust' $(4 \%)$. It is therefore possible to assert that this phase is characterized by a dominance of positive emotions.

Therefore, despite the new situation and the restrictive measures put in place to address the health crisis, the community, as mentioned above, reacted very consciously to the inevitable doubts without ever repressing any specific emotion unconsciously. From Fig. 4 it is possible to note how the Sentiment is well balanced, with positive feelings slightly advantageous 
for terms such as 'Drones', 'Police', 'Helicopter' and 'Control', and it is very biased towards positive feelings regarding the term 'security'. The response of the Sentiment is therefore in line with what was the emotional response during this phase. Such results suggest that this first phase will not leave unconscious emotional or sentimental aftermaths in the population.

Regarding phase 2, the distribution of every primary emotion is shown in Fig.5, while the ranking of the most cited words is shown in Fig.6.

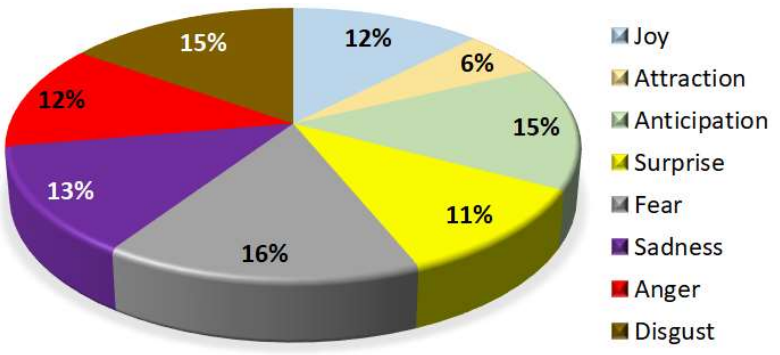

Fig. 5. Percentage distribution of every primary emotion for phase 2.

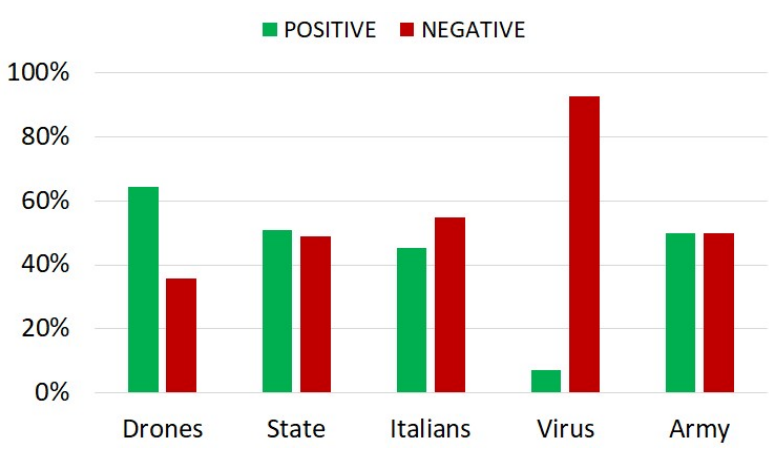

Fig. 6. Positive/negative ranking of the most cited Italian words (translated in English) for phase 2.

As it is possible to see from Fig.5, the dominant emotion occurring from the perception of safeness and risk of drones during phase 2 of the Coronavirus pandemic is 'Fear' $(16 \%)$, followed by 'Anticipation" (15\%) and 'Disgust' (15\%), 'Sadness' (13\%), 'Anger' $(12 \%)$ and 'Joy' (12\%), 'Surprise' (11\%), and 'Attraction' (6\%). It is therefore possible to assert that in this phase starts to be present a dominance of negative emotions.

If for the words 'State', 'Italians' and 'Army' there is a clear split in the middle of the community, it should be noted that the previously mentioned negative aftermath, conceivable for the term 'Virus', manifest themselves in the response of the Sentiment with greater strength, as it is possible to see in Fig.6. Recalling therefore that a sentiment represents a wellrooted trait of the soul, unlike emotions that have been fleeting moods, the term 'Virus' is certainly destined to leave an important negative trace in the minds of the community. If the emotion of 'Fear' was therefore predominant in the emotional response, partially hiding 'Anger', the latter is precisely the unconscious emotion responsible for the negative feelings that begin to show themselves decisively.
Regarding phase 3, the distribution of every primary emotion is shown in Fig.7, while the positive/negative ranking of the most cited words is shown in Fig.8.

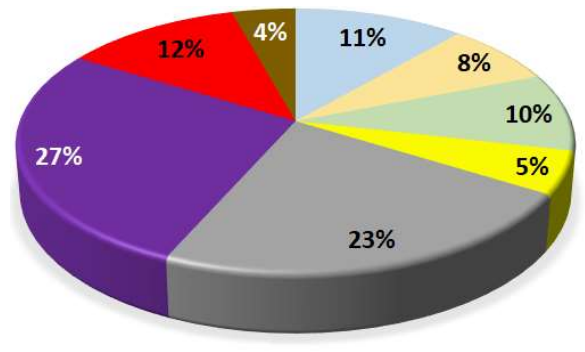

$$
\begin{aligned}
& \text { Joy } \\
& \text { Attraction } \\
& \text { Anticipation } \\
& \text { Surprise } \\
& \text { Aear } \\
& \text { Anger } \\
& \text { Disgust }
\end{aligned}
$$

Fig. 7. Percentage distribution of every primary emotion for phase

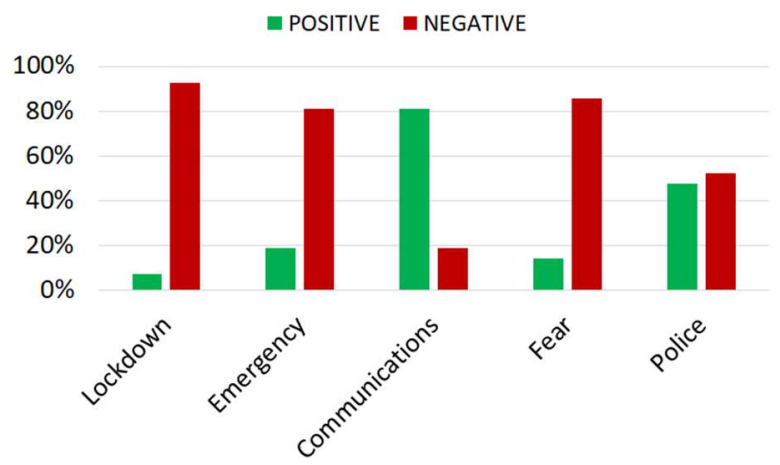

Fig. 8. Positive/negative ranking of the most cited Italian words (translated in English) for phase 3.

As it is possible to see from Fig.7, the dominant emotions occurring from the perception of safeness and risk of drones during Phase 3 of the Coronavirus pandemic is 'Sadness' $(27 \%)$, followed by 'Fear (23\%), 'Anger' (12\%), 'Joy' (11\%), 'Anticipation' (10\%), 'Attraction' (8\%), 'Surprise' (5\%), and 'Disgust' $(4 \%)$. It is therefore possible to affirm that this phase is characterized by a growth of negative emotions with respect to previous phase.

Fig.8 shows how the word 'Communications' is the only one to give rise to positive sentiments, also given its growing lack in daily life. It is anyway quite remarkable how the negative sentiment, which began to dominate in the second long phase, here it is practically predominant in an almost overwhelming way, especially for terms like 'Lockdown', 'Emergency' and 'Fear'. It can also be noted that the term 'Police', which at first gave rise to mostly positive feelings, now instead finds itself in a perfectly reversed situation, with negative feelings that surpass, albeit slightly, positive ones. So, just as the doubt had disappeared in the emotional response, also in the sentiment response there no longer appears to be any doubt: the community, in continuation of the trend undertaken in the second phase, increasingly and more strongly arouses negative sentiments, with 'Sadness' and 'Fear' emotions to be the masters, and thus presaging depressive effects on the community.

Regarding phase 4, the distribution of every primary emotion is shown in Fig.9, while the 
positive/negative ranking of the most cited words is shown in Fig.10.

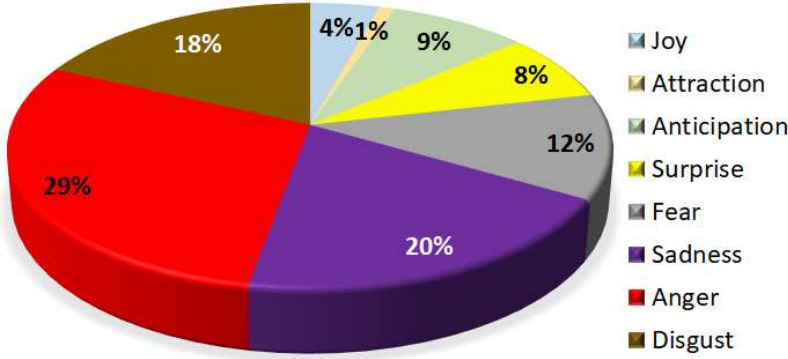

Fig. 9. Percentage distribution of every primary emotion for phase 4.

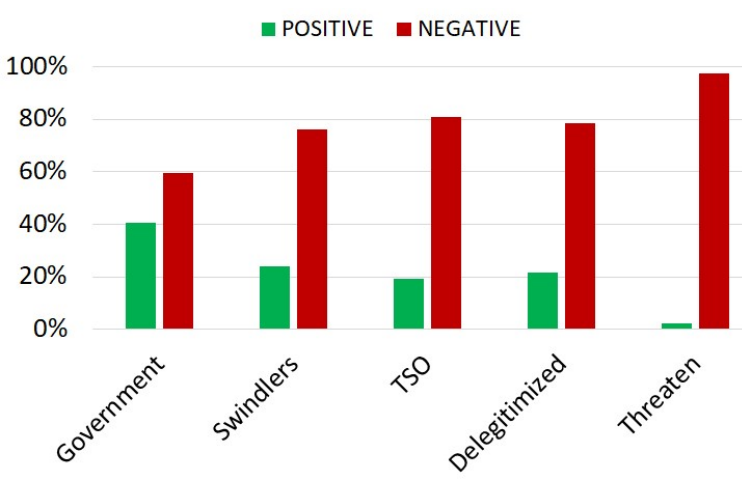

Fig. 10. Positive/negative ranking of the most cited Italian words (translated in English) for phase 4.

As it is possible to see from Fig.9, the dominant emotion occurring from the perception of safeness and risk of drones during Phase 3 of the Coronavirus pandemic is 'Anger' (29\%), followed by 'Sadness' (20\%), 'Disgust' (18\%), 'Fear (12\%), 'Anticipation' (9\%), 'Surprise' (8\%), 'Joy' (4\%), ‘Attraction' (1\%). It is therefore possible to affirm that this phase is characterized by a growth of negative emotions with respect to previous phase.

Fig. 10 shows how the length of this experience, which has been going on for several months, can leave a permanently negative trace within the community. There is no longer any doubt in people's emotional or sentimental experiences: from the results obtained it emerges that emotions such as 'Joy' and 'Attraction' have almost been forgotten. A behavior like this could let think that the character imprint that this experience will leave on people is profound and characterized by purely negative emotions.

The distribution of positive/negative primary emotions during the 4 phases is shown in Fig.11.

At it is possible to see from Fig.11, there is a remarkable trend of growth of negatives emotions and a significant reduction of positive emotions during the 4 phases. This means that there is no longer any doubt in people's emotional or sentimental experiences since it emerges, from the evaluation, that emotions such as 'Joy' and 'Attraction' have almost been forgotten in the last phase. A general trend like the considered one could let presume that the character imprint that these events will leave on population is deep and typified by entirely negative emotions.

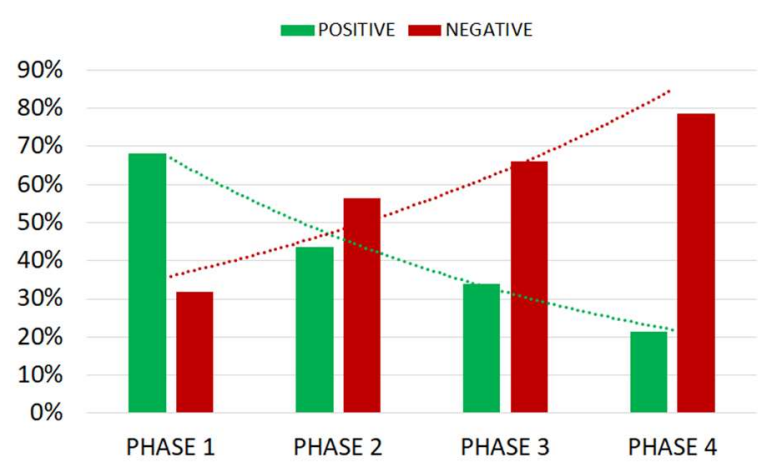

Fig. 11. Distribution of positive/negative primary emotions during the 4 phases.

Anyway, the fact that no emotion has been removed also indicates that the community has faced the issue consciously, albeit stressed by doubt.

The distribution of antithetical primary emotion of 'Joy' and 'Sadness', 'Anticipation' and 'Surprise, 'Attraction' and 'Disgust', 'Fear' and 'Anger' are shown in Figs. 12, 13, 14, 15 respectively.

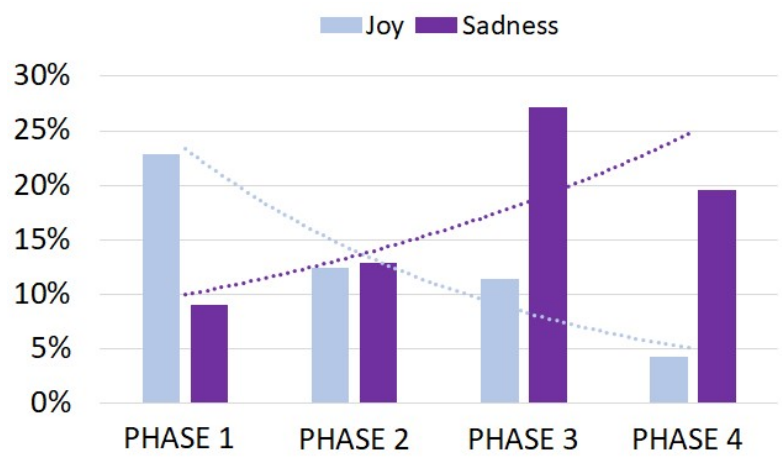

Fig. 12. Distribution of antitetichal primary emotions of 'Joy' and 'Sadness' during the 4 phases.

From Fig.12 it is possible to notice how the 'Joy''Sadness' couple of emotions has an inversely proportional trend. In fact, if initially 'Joy' was present and did not disappear completely in the second phase, but making itself matched by 'Sadness', the latter then takes a strong upper hand until the fourth phase, in which it dominates unchallenged with respect to 'Joy'.

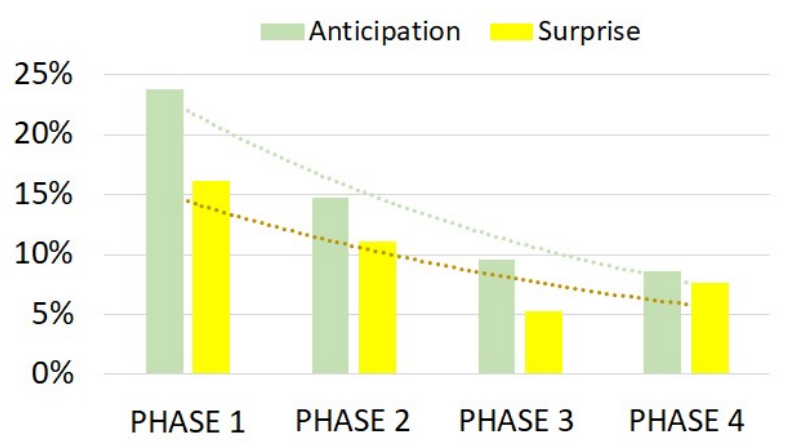

Fig. 13. Distribution of antitetichal primary emotions of 'Anticipation' and 'Surprise' during the 4 phases. 
From Fig.13 it is possible to notice that the emotions of 'Anticipation' and 'Surprise' always seem to be paired, thus indicating how they are among the main sources (whether large or small) of uncertainty in the community. However, they are primary positive emotions, and they are quite constantly lacking, even if with a trend not of free fall as is the case for other positive emotions.

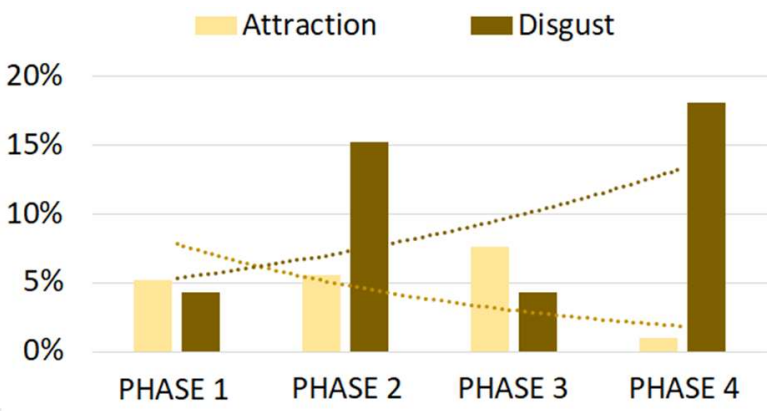

Fig. 14. Distribution of antitetichal primary emotions of 'Attraction' and 'Disgust' during the 4 phases.

Regarding the couple of emotions of 'Attraction' and 'Disgust' shown in Fig.14, there is a clear evidence of how the emotion of 'Disgust', very negative and not very present at the beginning, becomes predominant in the second phase and even more so in the fourth phase, where the 'Attraction' seems to be almost vanished. This suggests that many people, having almost removed this emotion, will be temporarily or definitively persons who are improbable to like something, which could be an object, a situation, or a person.

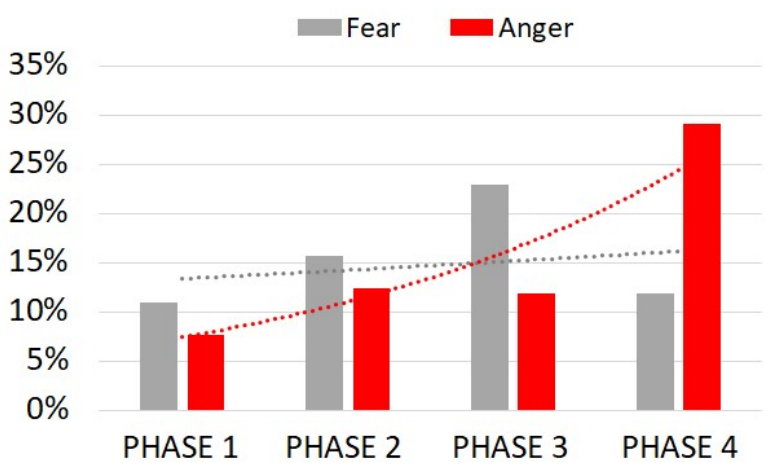

Fig. 15. Distribution of antitetichal primary emotions of 'Fear' and 'Anger' during the 4 phases.

Regarding Fig.15, it is possible to notice that the trend of the 'Fear' - 'Anger' couple highlights how these two emotions, purely negative, grow quite steadily over time, with the fourth phase characterized precisely by 'Anger' that dominates 'Fear'.

\section{CONCLUSIONS}

In the present work, a method for the evaluation of perception of safeness and risk of drones within during COVID-19 pandemic in Italy has been presented, granting value to the emotional aspects, through the semantic analysis of the textual contents present in Twitter.

It embodies a helpful method since it lets to have continuously opinions about risk perception in ordinary and critical contexts, such as the considered one.

\section{REFERENCES}

[1] F. Borghini, F. Garzia, G., Borghini, A. Borghini, The Psychology of Security, Emergency and Risk, WIT Press (UK and Boston - USA), 2016.

[2] W. Medhat, A. Hassan, A. H. Korashy, H., "Sentiment analysis algorithms and applications: A survey", Ain Shams Engineering Journal, 1093-1113, 2014.

[3] S. Ramalingam, "Metadata Extraction and Classification of YouTube Videos Using Sentiment Analysis" Proc. of IEEE International Carnahan Conference on Security Technology, Orlando (USA), 2016.

[4] E. Cambria, B. Schuller, Y. Xia, C. Havasi, "New Avenues in Opinion Mining and Sentiment Analysis", IEEE Intelligent Systems, Vol. 28, No.2, pp. 15-21, 2013.

[5] F. Garzia, R. Cusani, F. Borghini, B. Saltini, M. Lombardi, S. Ramalingam" "Perceived risk assessment through Open-Source Intelligent Techniques for Opinion Mining and Sentiment Analysis: the case study of the Papal Basilica and Sacred Convent of Saint Francis in Assisi, Italy", Proc. of IEEE International Carnahan Conference on Security Technologies, Montreal (Canada), pp. 11 - 15, 2018.

[6] F. Garzia, F. Borghini, A. Bruni, M. Lombardi, P. Mighetto, S. Ramalingam, S. B. Russo, "Emotional Reactions to the Perception of Risk in the Pompeii Archaeological Park", International Journal of Safety \& Security Engineering, IIETA (International Information and Engineering Technology Association), Vol.10, No.1, pp. 11 - 16, 2020.

[7] S. M. Park, D. K. Baik, Y. G. Kim, Y.-G. (2016). Sentiment User Profile Analysis based on Forgetting Curve in Mobile Environments. Proceedings IEEE 15th International Conference on Cognitive Informatics \& Cognitive Computing, pp. 207-211, 2016.

[8] S. Anastasia, I. Budi, "Twitter Sentiment Analysis of Online Transportation Service Providers" Proc. of International Conference on Advanced Computer Science and Information Systems, pp. 359-365, 2016.

[9] S. Akter, M. Aziz, "Sentiment Analysis On Facebook Group Using Lexicon Based Approach" Proc. of 3rd International Conference on Electrical Engineering and Information Communication Technology, pp. 1-4, 2016.

[10] Shaikh T., Deshpande D., "A review on Opinion Mining and Sentiment Analysis", International Journal of Computer Applications (0975-8887), 2016

[11] E. Mayor, L. M. Bietti, "Twitter, time and emotions", R. Soc. Open Sci. 8: 201900, 2021.

[12] A. Mathur, P. Kubde and S. Vaidya, "Emotional Analysis using Twitter Data during Pandemic Situation: COVID-19", 2020 5th International Conference on Communication and Electronics Systems (ICCES), pp. 845-848, 2020.

[13] S. Sharma and A. Sharma, "Twitter Sentiment Analysis During Unlock Period of COVID-19", 2020 Sixth International Conference on Parallel, Distributed and Grid Computing (PDGC), pp. 221-224, 2020.

[14] A. Kumar, S. U. Khan, A. Kalra, "COVID-19 pandemic: a sentiment analysis: A short review of the emotional effects produced by social media posts during this global crisis", European Heart Journal, Vol.41, No.39, pp.3782-3783, 2020.

[15] A. Mathur, P. Kubde and S. Vaidya, "Emotional Analysis using Twitter Data during Pandemic Situation: COVID-19", 2020 5th International Conference on Communication and Electronics Systems (ICCES), pp. 845-848, 2020.

[16] J. Samuel et al, "Feeling Positive About Reopening? New Normal Scenarios From COVID-19 US Reopen Sentiment Analytics", in IEEE Access, vol. 8, pp. 142173-142190, 2020. 